\title{
Annotations
}

\section{DNA markers and Duchenne muscular dystrophy}

The localisation of the gene for Duchenne muscular dystrophy using DNA polymorphisms has been one of the most exciting episodes in muscular dystrophy research and marks the first clinical application of these polymorphisms to a genetic disorder where the primary gene product is unknown. Previously the main applications of the new DNA technology had been to identify genes, such as those for the haemoglobinopathies, whose function was already well understood; the possibility of using allied techniques for those numerous disorders about which little or nothing except their Mendelian inheritance is known, is particularly important clinically, since tests of predicition and prevention are usually lacking or inadequate.

The paper by Dr Pembrey and colleagues in this issue of Archives is likely to be the first of many applying DNA polymorphisms to genetic disorders, and some of the concepts and terminology will be unfamiliar to paediatricians, just as were the symbols used in the early days of cytogenetics. It is worth explaining some of the main terms here.

The term polymorphism, meaning an inherited variation where two or more forms are seen with reasonable frequency, will be familiar from blood groups and protein characteristics. Restriction fragment length polymorphism (RFLP) refers to the variably sized DNA fragments produced when DNA is cut with a specific restriction enzyme. The polymorphism is shown up as different positions of bands on electrophoresis (see Fig. 1 in the Pembrey paper), the smaller fragments travelling faster than the larger. For convenience and stability the DNA is usually transferred to a nitrocellulose filter by the process known as Southern blotting.

To show up such a polymorphism among the myriad fragments of DNA in a sample from white blood cells (or chorionic villus) requires a gene probe. This is a specific DNA sequence which will seek out and bind to any corresponding sequence present in the patient sample on the filter; since it is radiolabelled it will produce a band or bands when a radiograph is exposed against the filter. Gene probes can be produced from various sources and if the gene product is known, it may be possible to construct a specific $c D N A$ probe. If not (as in Duchenne muscular dystrophy), one has to use
DNA sequences selected from collections of DNA (DNA libraries); these may be derived from $\mathrm{X}$ chromosome specific DNA (for example probe $\mathrm{RC}_{8}$ ) or from total human DNA (probe L1.28).

Returning to the use of these techniques in the muscular dystrophies; Duchenne muscular dystrophy was first found to be linked to probe $\mathrm{RC}_{8}$, with around $15 \%$ recombination between the two loci; ${ }^{1}$ later probe L1.28 was found to be a similar distance on the opposite side (Figure) ${ }^{2}$ Similar studies on families with Becker muscular dystrophy have found that, contrary to previous thinking, it shows similar linkage relations to Duchenne muscular dystrophy, and may well be allelic with it. ${ }^{3}$

Thus the Duchenne (and Becker) muscular dystrophy genes have now been pinned down to a restricted area of the $\mathrm{X}$ chromosome and current research is focusing on means of identifying the actual mutational defects. This may prove a difficult task, though if a proportion of cases results from an actual deletion of the gene, as has been shown for some thalassaemias and some cases of haemophilia $\mathrm{B},{ }^{4}$ these should be readily detectable by a DNA probe that includes the disease locus.

The present markers, each with a built in error rate in prediction of at least $15 \%$ because of recombination between marker and disease, are not ideal for clinical application, but with care and using them in combination with currently available tests, they can be of real help to families. Pembrey et al estimated that 69 of 70 families in their series could be helped to some degree, and selected examples of clinical use have already been reported..$^{5}$

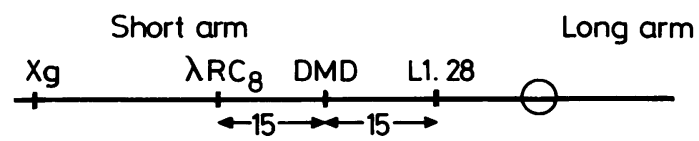

Figure Schematic map of the short arm of the X chromosome showing the approximate location of the Duchenne muscular dystrophy locus $(D M D)$ in relation to two $D N A$ markers. The locus for Becker muscular dystrophy is similar or identical. 
Where both marker sytems are informative an accurate prediction can be given, since if no recombination is found between the two markers, it would require the unlikely event of a double crossover to give an error in prediction for Duchenne muscular dystrophy. More commonly only one marker will give information, and here the value of DNA tests will usually be in combination with creatine kinase testing which may produce a definitive prediction that would not have resulted from the use of either approach on its own.

The detailed example given by Pembrey et al illustrates both the advantages and the current limitations of DNA techniques in Duchenne muscular dystrophy. The prediction for heterozygotes is unaffected by pregnancy or age (unlike creatine kinase) and there is no problem of variation from $\mathrm{X}$ chromosome inactivation to contend with. Prenatal use of DNA markers in fetal tissue from chorion biopsy has already been validated for the probes in question, ${ }^{6}$ and is already coming into clinical use for first trimester fetal sexing, though the safety of chorion biopsy remains to be established. The example also illustrates, however, some of the disadvantages of the use of linked polymorphisms: the risk calculations may seem formidable and the modification in the final risk is not always great.

How should the clinician working with Duchenne muscular dystrophy patients react to these new developments-by taking blood for DNA analysis from all the families under his or her care, or by waiting until a really close marker is available that will give an accurate prediction in all circumstances? Personal experience suggests a few guidelines:

(1) Blood should be taken for DNA isolation from any boy with Duchenne muscular dystrophy likely to die in the near future, so that the opportunity to utilise the approach in future for relatives is not irretrievably lost.

(2) Where there is a number of affected members in a family, particularly in more than one sibship, serious consideration should be given to typing the kindred as a whole, including relevant spouses and healthy males. Such a family is most likely to provide clear cut predictions for carriers.
(3) Isolated cases are rarely likely to give helpful information at present but in the future closer markers will allow a valuable normal prediction by showing that a second child has not inherited the same maternal gene as was transmitted to a previous affected child.

(4) Prenatal use should await closer markers except in the few families where both probes give information.

Meanwhile, research developments that will identify the Duchenne muscular dystrophy gene itself are eagerly awaited and, given the rapidity of developments so far and the intense interest in the subject by molecular biologists as well as clinicians, this now seems a very real prospect for the near future.

\section{References}

${ }^{1}$ Murray JM, Davies KE, Harper PS, Meredith L, Mueller CR, Williamson R. Linkage relationship of a cloned DNA sequence on the short arm of the $X$ chromosome to Duchenne muscular dystrophy. Nature 1982;300:69-71.

2 Davies KE, Pearson PL, Harper PS, et al. Linkage analysis of two cloned DNA sequences flanking the Duchenne muscular dystrophy locus on the short arm of the human X chromosome. Nucleic Acids Res 1983;11:2303-12.

${ }^{3}$ Kingston HM, Thomas NST, Pearson PL, Sarfarazi M, Harper PS. Genetic linkage between Becker muscular dystrophy and a polymorphic DNA sequence on the short arm of the $\mathrm{X}$ chromosome. J Med Genet 1983;20:255-8.

${ }^{4}$ Giannelli F, Choo KH, Rees DJG, Boyd Y, Rizza CR, Brownlee GG. Gene deletions in patients with haemophilia B + anti-factor IX antibodies. Nature 1983;303:181-2.

5 Harper PS, O'Brien T. Murray JM, Davies KE, Pearson P, Williamson $R$. The use of linked DNA polymorphisms for genotype prediction in families with Duchenne muscular dystrophy. J Med Genet 1983;20:252-4.

6 Elles RG, Wiliamson R, Niazi M, Coleman DV, Horwell D. Absence of maternal contamination of chorionic villi used for fetal gene analysis. $N$ Engl $J$ Med 1983;368:1433-5.

$$
\begin{array}{r}
\text { P S HARPER } \\
\text { Section of Medical Genetics, } \\
\text { Department of Medicine, } \\
\text { Welsh National School of Medicine, } \\
\text { Heath Park, } \\
\text { Cardiff CF4 } 4 X N
\end{array}
$$

